\title{
Modeling Epac1 interactions with the allosteric inhibitor AM-001 by co-solvent molecular dynamics
}

\author{
Marianna Bufano ${ }^{1}$ (D) Marion Laudette ${ }^{2,3}$ (D) Jean-Paul Blondeau ${ }^{4}$ (D) Frank Lezoualc' $\mathbf{h}^{2,3}$ (D) Marianna Nalli ${ }^{1}$ (D) . \\ Romano Silvestri $^{1}$ (D) Andrea Brancale ${ }^{5}$ (D) . Antonio Coluccia ${ }^{1}$ (D)
}

Received: 25 February 2020 / Accepted: 13 July 2020 / Published online: 22 July 2020

(c) The Author(s) 2020

\begin{abstract}
The exchange proteins activated by cAMP (EPAC) are implicated in a large variety of physiological processes and they are considered as promising targets for a wide range of therapeutic applications. Several recent reports provided evidence for the therapeutic effectiveness of the inhibiting EPAC1 activity cardiac diseases. In that context, we recently characterized a selective EPAC1 antagonist named AM-001. This compound was featured by a non-competitive mechanism of action but the localization of its allosteric site to EPAC1 structure has yet to be investigated. Therefore, we performed cosolvent molecular dynamics with the aim to identify a suitable allosteric binding site. Then, the docking and molecular dynamics were used to determine the binding of the AM-001 to the regions highlighted by cosolvent molecular dynamics for EPAC1. These analyses led us to the identification of a suitable allosteric AM-001 binding pocket at EPAC1. As a model validation, we also evaluated the binding poses of the available AM-001 analogues, with a different biological potency. Finally, the complex EPAC1 with AM-001 bound at the putative allosteric site was further refined by molecular dynamics. The principal component analysis led us to identify the protein motion that resulted in an inactive like conformation upon the allosteric inhibitor binding.
\end{abstract}

Keywords EPAC $\cdot$ Molecular Dynamics $\cdot$ Cosolvent Molecular Dynamics $\cdot$ Docking $\cdot$ PCA

Electronic supplementary material The online version of this article (https://doi.org/10.1007/s10822-020-00332-y) contains supplementary material, which is available to authorized users.

Antonio Coluccia

antonio.coluccia@uniroma1.it

1 Department of Drug Chemistry and Technologies, Istituto Pasteur Italia - Fondazione Cenci Bolognetti, Sapienza University of Rome, Piazzale Aldo Moro 5, 00185 Rome, Italy

2 Institut des Maladies Métaboliques et Cardiovasculaires, INSERM UMR-1048, Cedex 04, 31432 Toulouse, France

3 Université de Toulouse - Paul Sabatier, Cedex 04, 31432 Toulouse, France

4 Faculté de Pharmacie, Université Paris-Sud, Châtenay-Malabry Cedex, 92296 Paris, France

5 Cardiff School of Pharmacy and Pharmaceutical Sciences, King Edward VII Avenue, Cardiff CF103NB, UK

\section{Introduction}

The cyclic adenosine monophosphate (cAMP) is a universal second messenger that regulates many biological processes, including cell proliferation, differentiation, and apoptosis [1]. The effects of cAMP in mammalian cells are mediated by at least three effector families: protein kinase A (PKA), exchange proteins activated by cAMP (EPAC) and ion channels bearing a cyclic nucleotide binding domains (CNBD). The EPAC proteins regulate a variety of physiological processes, such as calcium homeostasis in cardiomyocytes, insulin secretion from pancreatic $\beta$ cells, integrin-mediated cell adhesion, and cell death [2]. Therefore, EPAC proteins are considered as targets for a wide range of therapeutic applications [3]. To date, two EPAC isoforms have been identified, EPAC1 and EPAC2 which display distinct pattern of tissue expression. Indeed, EPAC1 is ubiquitously expressed whereas EPAC2 and its slice variants are localized in the brain (EPAC2A), pancreatic cells (EPAC2B) testis and liver (EPAC2C) [4].

EPAC1 and EPAC2 are multidomain proteins featured by an $\mathrm{N}$-terminal regulatory region $(\mathrm{RR})$ and a $\mathrm{C}$-terminal 
catalytic region (CR). The amino-terminal regulation region contains a Disheveled/Egl-10/pleckstrin (DEP) domain followed by a cyclic nucleotide-binding domain (CNBD). EPAC2A has an additional low-affinity CNBD, which is unable to induce guanine nucleotide exchange factor (GEF) activity after cAMP binding [5]. The catalytic region consists of a Ras exchange motif (REM), a Ras association domain (RA) and a CDC25 homology domain (CDC25HD). The CDC25HD catalyses GDP-GTP exchange for Rap, while the REM domain contributes to stabilize a catalytic helix, and the RA domain influences EPAC subcellular localization [6]. The regulatory CNBD at the RR C-terminus [7, 8] controls the relative orientations of the $\mathrm{RR}$ and $\mathrm{CR}$ with respect to each other. In the absence of cAMP, the two regions adopt a closed conformation, in which the RR restricts access of the Rap GTPases to the CR, resulting in constitutive inhibition. Upon binding of cAMP to the conserved CNBD, the two regions adopt an open (active) topology, where the Rap substrate can interact with the CR to promote GDP-GTP exchange [7-10] (Fig. 1).

In the heart, cAMP represents one of the most important mechanism for increasing cardiac function in response to acute sympathetic stimulation of $\beta$-adrenergic receptor $(\beta$ -AR) [11]. Although acute activation of $b-A R$ has beneficial effect on cardiac function, chronic b-AR stimulation of this pathway promotes pathological cardiac remodelling, which may ultimately lead to heart failure (HF) [12]. Importantly, recent data show pharmacological or genetic inhibition of EPAC1 prevents cardiac hypertrophy and fibrosis induced by sustained $\beta$-AR activation and improves cardiac function [13].

Therefore, targeting EPAC1 may have potential therapeutic benefits in cardiac diseases [13, 14] (Fig. 1).

Currently, there are only few EPAC1 modulators [14]. Based on their chemical structure these compounds may be divided in cAMP analogue and non-nucleotidic small molecules. The major drawbacks about the reported modulators were the lack of selectivity between the EPAC isoforms or PKA, and the unsuitable drug-like properties. Another concern about modulator of cAMP-regulated protein is that the orthosteric cAMP site is amply conserved making selectivity a crucial issue [15]. This task may be solved looking for non-competitive allosteric modulator that is expected to have reduced risks of cross-reactivity with other receptors that signal via cAMP such as PKA or CNG ion channels [16]. To our knowledge there are just two compounds, CE3F4 [17, 18] and AM-001 [13], that are able to selectively inhibit EPAC1. The small molecule AM-001 is a thieno[2,3-b]pyridine derivative (Table S1) acting as a selective non-competitive antagonist of EPAC1 [13]. AM-001 mitigates cardiac hypertrophy, inflammation and fibrosis, and improves cardiac function during chronic $\beta$-adrenergic receptor activation. In in-vivo experiments, AM-001 reduces the infarct size after mouse myocardial ischaemia/reperfusion injury [13].

Since the biological evaluation suggested a non-competitive mechanism of action for the AM-001 derivative [13] we sought to identify its putative allosteric binding site. To date, there are not experimental data about the allosteric site localization. It was just reported that the hinge region of EPAC might be a druggable region because of its crucial role in the close to open conformation transition $[19,20]$.

The cosolvent molecular dynamics (CMD) method is very useful to highlight both catalytic and allosteric sites [21]. This method was inspired by the multiple solvent crystal structures (MSCS) technique [22]. The MSCS is based on solving crystal structures of proteins in the presence of various organics cosolvents. Overlapping locations of different cosolvents were found to be highly correlated with regions of biological significance [21]. The CMD could be considered the in-silico counterpart of the MSCS experiments. The CMD method uses the molecular dynamics simulation of a protein solvated by an organic solvents/water mixtures to
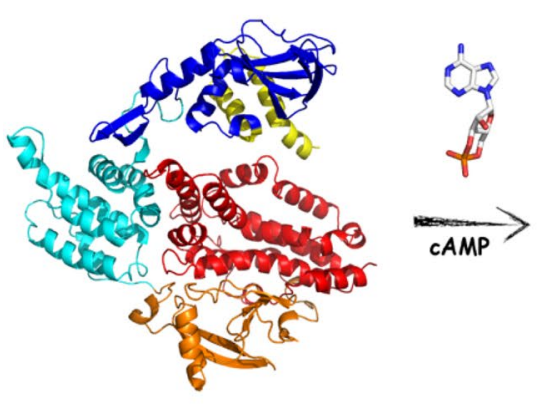

Fig. 1 Graphical representation of EPAC activation upon cAMP binding. CNBD, DEP, REM, RA, and CDC25-HD domains are coloured in yellow, blue, cyan, orange and red respectively. cAMP is reported as white stick, RAP is reported as green cartoon. The cyclic nucleotide binding allows the regulatory domain to open leading the catalytic region exposed for binding of Rap 
determine the sites where the organics solvents preferentially bind.

In our work, we attempted to identify the EPAC1 allosteric binding site by using CMD. Thus, the EPAC1 active and inactive conformations were solvated with a solution of $20 \%$ (w/w) of isopropanol (ISO), ethanol (ETOH), and dimethylsulfoxide (DMSO), in water, then the cosolvent occupancy maps were generated and investigated.

ISO is the most used solvent for CMD as it is able to interact with hydrophobic protein sites [23]. DMSO and ETOH are chosen for their hydrogen-bond acceptor and donor capabilities respectively [24, 25]. The cosolvents were also chosen to be fully water miscible to avoid molecules aggregations phenomena and the concentration was fixed to avoid protein denaturation [26].

The models of the inactive and active EPAC1 conformations were generated by homology from the EPAC2 crystal structure (inactive state: PDB code 2BYV [27]; active state PDB code 3CF6 [6]).

To evaluate that the unfolding phenomena did not affect the protein during the simulations the RMSD of the backbone $C \alpha$ was computed [28]. The RMSD values showed that cosolvents molecules did not disturb the protein folding (Figs. S1, S2).

The trajectory of each simulation was analysed to determine the cosolvent occupancy maps. Each map indicates the pockets where the cosolvent molecules were most frequently located. The interaction energy among protein and cosolvent molecules was not taken into account. A size cutoff was applied to remove the smaller disconnected part of the maps doing interpretation easier. Thus, the protein areas where the cosolvent occupancy maps were superimposable, represented the suitable sites for a partner binding (Figs. 2 and 3 and S3, S4).

\section{Materials and methods}

\section{EPAC structures}

The EPAC structure was downloaded from the PDB data bank (http://www.rcsb.org/), inactive state: PDB code 2BYV; [27] active state PDB code 3CF6 [6].

Homology model were carried out by homology model PRIME [29] of MAESTRO [30]. The obtained models were examined to avoid steric clashes, bond length deviations and angles, etc., by using the Ramachandran plot and the Protein Preparation Wizard. [31] The missing loops were obtained by Raptor X $[32,33]$. The primary sequence of EPAC1 and EPAC2 was downloaded by UniProtKB (https://www.unipr ot.org) code: O95398NCBI and UniProtKB code: Q8WZA2 respectively.

\section{Molecular dynamics}

Molecular dynamics was performed with the AMBER 12 suite [34]. For simulation in water the protein was solvated in a periodic octahedron simulation box using TIP3P water molecules, providing a minimum of $10 \AA$ of water between the protein surface and any periodic box edge. The organic cosolvent/water box were prepared by packmol by fixing a $20 \% \mathrm{w} / \mathrm{w}$ ratio [35]. Parameters for cosolvents were obtained by pyMDMix. (http://mdmix.sourceforge.net) Then, ions
Fig. 2 Epac1 active conformation cosolvent occupancy maps selected areas. Epac1 is reported as cartoon: CNBD and DEP green; REM orange; CDC25-HD blue and RA red. Yellow maps are for ETA; cyan for ISO and pink for DMSO

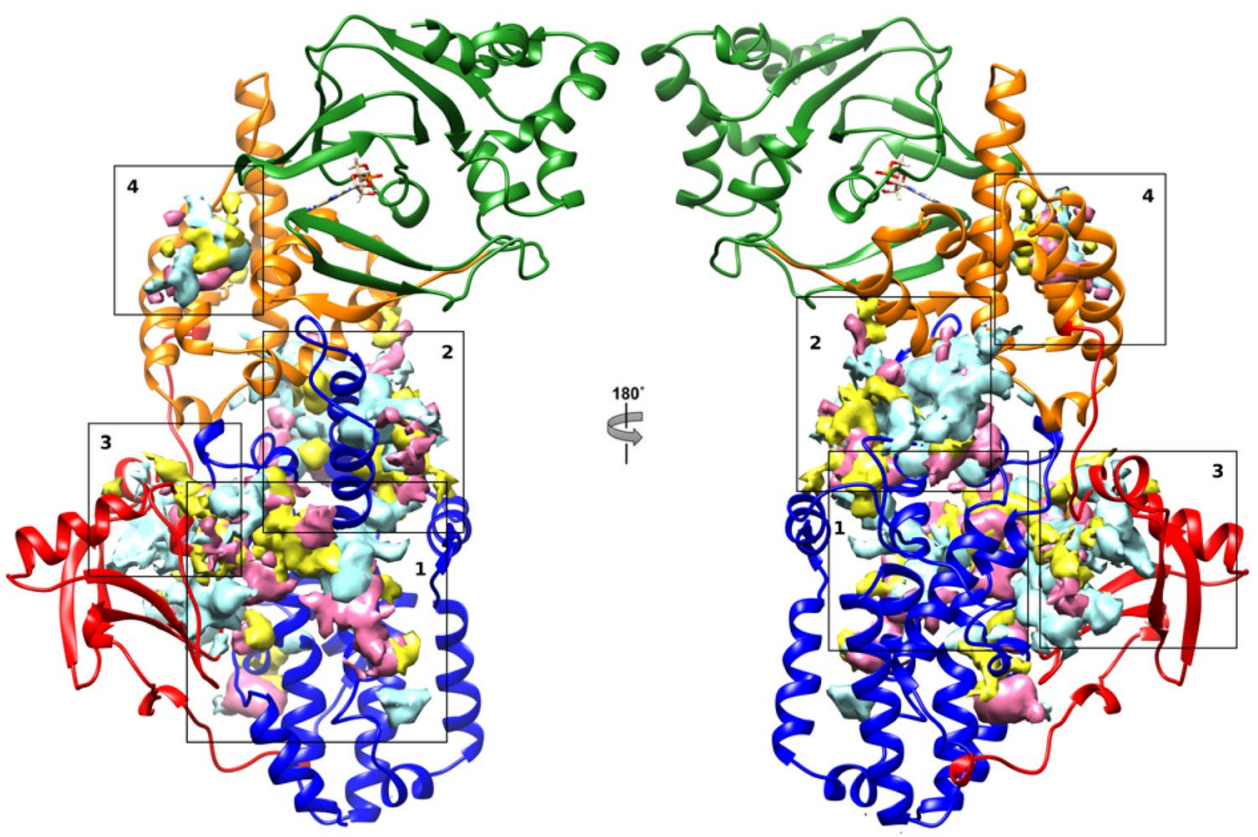




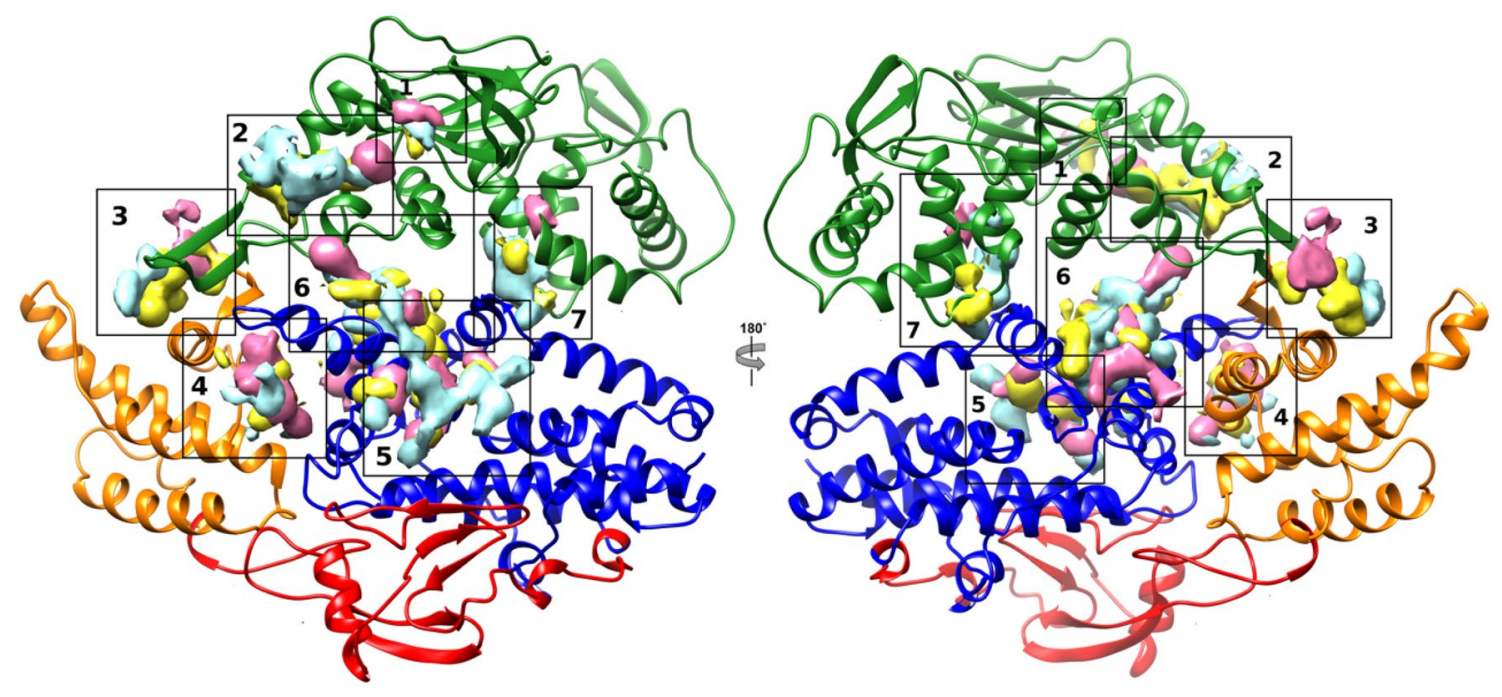

Fig. 3 Epac1 inactive conformation cosolvent occupancy maps selected zones. Epac1 inactive conformation isreported as cartoon: CNBD and DEP green; REM orange; CDC25-HD blue and RA red. Yellow maps are forETA; cyan for ISO and pink for DMSO

were added to neutralize the charge of the total system. The solvents molecules and the ions were energy-minimized keeping the coordinates of the protein-ligand complex fixed (1000 cycle), and then the whole system was minimized (5000 cycle). Following minimization, the entire system was heated to $298 \mathrm{~K}$ ( $20 \mathrm{ps}$ ). The production simulation was conducted at $298 \mathrm{~K}$ with constant pressure and periodic boundary condition. Shake bond length condition was used $($ ntc $=2)$. Production was carried out on GeForce gtx 780 gpu. The cosolvent occupancy maps were computed with trajectories length of $100 \mathrm{~ns}$ and $250 \mathrm{~ns}$. The obtained maps were similar regardless the length of the trajectories.

The production length of the simulations to evaluate the AM-001 stability at the studied sites was $10 \mathrm{~ns}$. The production time to run PCA was $200 \mathrm{~ns}$. All simulations were repeated five times. The occupancy maps were calculated by Chimera Volume viewer [36]. Chimera we also used for the representation of the maps by volume viewer range $50 \%$, and for the size filtering by the Hide Dust tool size 5.0 [37]. Compounds were parametrized by Antechamber [38, 39] using BCC charges. Trajectories analysis were carried out by cpptraj program [40].

\section{PCA}

The PCA analyses were carried out by cpptraj program [40]. The trajectory was firstly superimposed to an average structure obtained by cpptraj. Then, the trajectory of the a-carbon was extracted and the covariance matrix calculated. The diagonalization of the covariance matrix generated a set of eigenvectors that gave a vectorial description of the motion. The computed eigenvectors and eigenvalues were used to draw the porcupine plot by means of the Normal
Mode Wizard module (NMWiz) [41, 42] of the VMD gui [43].

\section{Molecular docking}

Ligand structures were built with MAESTRO [30] and minimized using the OPLS3 force field until a rmsd gradient of $0.05 \mathrm{kcal} /(\mathrm{mol} \AA)$ was reached. The docking simulations were performed using Gold, [44] Plants [45] and Autodock [46]. We set a binding lattice of radius or side large enough to cover the whole studied maps, then all default settings were used. The Docking of the AM-001 analogues were carried out by Plants [45], using as receptor a representative structure obtained by the trajectory of the EPAC1/posa3_6. The representative structure was the average structure extracted by cpptraj [40] after a stable rmsd was reached. The poses binding free energy was calculated by MAESTRO prime MMGB-SA module [47]. The pictures reported in the manuscript were done with Pymol [48].

\section{Results and discussion}

\section{Analyses of active conformation occupancy maps}

The active conformation of the enzyme was mainly studied to access the quality of the method. The inhibition of EPAC1 by AM-001 was previously determined by BRET assays [17]. Indeed, the experiment measured the level of activation of the EPAC1, namely the shift from inactive to active form of the enzyme after the antagonist binding. The cosolvent occupancy maps were computed with trajectories 
of $100 \mathrm{~ns}$ (Fig. S3) and $250 \mathrm{~ns}$ (data not shown). The occupancy maps analyses for the EPAC1 active conformation led to the identification of four interesting zones signed as areas from 1 to 4 (Fig. 2) regardless of the trajectories length.

The areas 1 and 2 were located at the CDC25-HD domain and corresponded to the Rap binding site [6]. Indeed, the superimposition of the EPAC2 crystal structure (PDB code: 4MGI) [49], bearing Rap interacting domain, with the EPAC1 model clearly showed that the areas 1 and 2 were filled by this domain. Specifically, we observed that the area 1 involved $4 \alpha$ helices of the CDC25-HD namely $\alpha 1$ (671-680), $\alpha 3$ (708-713), $\alpha 5$ (750-756) and $\alpha 9$ (839-844). This area bound the $\beta 4$ and the loop linking $\beta 4$ to $\alpha 3$ of the Rap domain. (Fig. S5) The area 2 involved the CDC25 Helical Hairpin ( $\alpha 9$ and $\alpha 10$ from 823 to 854 ) that accommodated the loop between $\alpha 1$ and $\beta 2$ of Rap [50]. (Fig. S5) The area 2 was also reported to be potentially involved in an interaction between EPAC1 and RanPB2 [51].

The site 3 was located at the interface between the CDC25-HD and RA domains, and comprised a loop absent in EPAC2. This broad surface encompassed part of the loop (550-557), the CDC25-HD $\alpha 1$ (678-682) and the $\beta 1$ (558-562) of RA domain. This area was reported to be suitable for a protein-protein interaction [52].

The area 4 was located at the REM domain. It comprised the $\alpha 1$ (394-398) and $\alpha 3$ (434-440) helices of REM and a small loop of CNBD (294-299). This zone was identified to be suitable for partners binding by FTPmap experiments [20].

Various proteins with undefined binding pockets, contributed to the regulation and/or the subcellular localization of EPAC1 [51]. Indeed, it was reported that the residues 838-881 were involved in the compartmentalization of EPAC1, but it was not clarified how these residues drove this activity [52]. Furthermore, tubulin was also reported as a direct partner of EPAC1, but its binding site was not yet identified [53]. Our analyses of the occupancy maps suggested that some areas were suitable for binding of partner proteins are consistent with the available structural data, validating the appropriateness of the method used.

\section{Occupancy maps analyses of inactive conformation}

The CMD procedure was then applied to the EPAC1 autoinhibited form (inactive conformation) with the aim to identify a putative binding site for AM-001. The cosolvent occupancy maps were computed by trajectories of $100 \mathrm{~ns}$ (Fig. 3) and $250 \mathrm{~ns}$ (Fig. S6). The analyses of the cosolvent occupancy maps did not show any significant differences depending on the trajectories length and led to the identification of some attractive areas. The proposed mechanism of cAMP-induced EPAC1 activation involves EPAC1 regulatory domain that moves away from the catalytic domain. In this transition a crucial role is played by the lid (first $\beta$-strand of REM 384-391 and the tip of CDC25-HD HP 832-837) and the hinge helix (CNBD 299-308). Accordingly, AM-001 binding to its allosteric pocket should have to impair this cAMP-dependent conformational change [20]. These data were used to reject the area located at the CDC25-HD and the RA domains. Indeed, the areas appear to be too far from both the lid and hinge helix to influence the inactive to active conformation state. This observation led us to managed 7 areas (Fig. 3).

Area 1 was the cAMP binding pocket [6]. The identification of the cAMP site further validated the reliability of the method.

The remaining areas were inspected by docking and molecular dynamics. Area 2 was located at the CNBD close to the catalytic site and it encompassed CNDB $\alpha 3$ and the following loop (245-250), $\alpha 4$ (251-260) and $\alpha 6$ (344-350). Area 3 was at the interface between CNBD and REM domains. This site is delimited by REM $\beta 1$ (387-392) and $\alpha 1$ (398-402) together with the CNBD $\beta 9$ (353-359) and $\beta 10$ (363-368). Area 4 involved the HP loop of CDC25-HD and the REM $\alpha 2$ (423-430). Area 5, was the only one entirely located at the CDC25-HD comprising the $\alpha 2$ (689-692), $\alpha 3$ (704-708), $\alpha 5$ (761-765) and $\alpha 9$ (815-827). The area 6 was at the interface of CDCD25-HD and REM. The area was defined by the $\alpha 8$ (800-805), $\alpha 9(817-822)$ and $\alpha 10$ (844-851) of CDC25-HD and the loop between $\alpha 2$ and $\alpha 3$ (247-251) of the CNDB. Lastly, area 7 was located between the CDC25-HD and the CNDB domains involving the $\alpha 8$ (793-801) of the CDC25-HD and the long helix (206-216) linking CNDB to the Dep domain. (Fig. S7)

Thus, AM-001 was docked to each of the listed areas. The docking studies were carried out with Gold, [44] Plants [45] and Autodock [46].

For each area, at least one consistent AM-001 binding pose (RMSD $<1.5 \AA$ ) among the docking software was selected and submitted to molecular dynamic simulations (10 ns).

For the areas 2, 3, 4 and 7 the analyses of the molecular dynamic trajectories showed that the ligand moved out of the pocket at the early stage of the simulation. In fact, these sites were markedly solvent exposed. This feature was not common for small molecules binding site $[54,55]$, and it was therefore hypothesized that these sites were more appropriate as protein-protein interaction pocket [56, 57].

Then we managed the two remaining areas, namely 5 and 6. Once again, the selected binding poses were submitted to molecular dynamics simulations. The trajectories analysis showed two stable $(\mathrm{rmsf}<1.0 \AA$ ) binding modes for area 5 (termed as pose4_5 and pose9_5) (Figs. S9, S10) and one for area 6 (termed as pose3_6).

Since AM-001 preferentially bound to the EPAC1 isoforms $\left(\mathrm{IC}_{50 \mathrm{EPAC} 1} 48.5 \mu \mathrm{M}\right.$ ), and it was ineffective in 
suppressing EPAC2 activity $\left(\mathrm{IC}_{50 \mathrm{EPAC} 2}>1000 \mu \mathrm{M}\right)[13]$ we investigated whether the 3 selected binding mode might be suitable for EPAC2. Thus, we carried out a series of molecular dynamics simulations of the 3 binding modes placed in the corresponding pockets of EPAC2. The trajectories and the binding free energy were calculated [58, 59] and compared with those resulting from the EPAC1 simulations.

The trajectories inspection showed that the poses for area 5 were stable for EPAC2 (pose4_5 rmsf 0.49 A pose9_5 rmsf $0.39 \AA$ ) as observed for EPAC1 (pose4_5 rmsf $0.41 \AA$ pose9_5 rmsf $0.60 \AA$ ). The computed binding free energies for EPAC1 and EPAC2 were very similar (EPAC1 $\Delta \mathrm{G}$ pose $4 \_5=-53.9$, pose9 $-5=-56.1 ;$ EPAC2 $\Delta$ G pose4_5 $=-63.3$ pose9 $5=-53.1)$. The difference was just 3 $\mathrm{Kcal} / \mathrm{mol}$ for pose9_5, and a difference of $10 \mathrm{Kcal} / \mathrm{mol}$ for pose4_5 with the best $\Delta \mathrm{G}$ value for EPAC2.

These data could indicate that area 5 is not be suitable for binding of AM-001 as a selective allosteric inhibition of EPAC1. This site could however correspond to a region of biological significance for the enzymatic activity of both EPAC isoforms.

The same analysis was repeated also for the area 6 pose (pose3_6). The complex EPAC2/pose3_6 was submitted to molecular dynamic. The trajectory inspection clearly showed a level of instability. The compound moved toward the solvent, showing a rmsd higher than $3 \AA$ and a calculated $\Delta \mathrm{G}$ of $-45.9 \mathrm{kcal} / \mathrm{mol}$. The complex EPAC1/pose3_6 trajectory was inspected in the same way. The AM- 001 pose showed a general stability with a rmsd of $1.19 \AA$ and a calculated $\Delta \mathrm{G}$ of $-65.1 \mathrm{kcal} / \mathrm{mol}$, thus with about $20 \mathrm{kcal} / \mathrm{mol}$ in favour of the EPAC1 binding.

These data suggested that area 6 might be the most suitable for the EPAC1 allosteric inhibition. To validate our idea, we extended the molecular dynamic simulation to $200 \mathrm{~ns}$ and we testes the ability of this binding site to match known SARs profile of the compound analogues (Table S1).

The analyses of the extended trajectory showed that the selected binding mode was stable and led us to identify a series of pharmacophoric interactions: the fluorinated phenyl group had a pi-cation interaction with R801 side chain and hydrophobic contacts with L235 and M; the primary amine moiety as well as the amidic nitrogen atom were involved in H-bond with D234 side chain; the pyridine thiophene fused ring had hydrophobic contacts with F237, R255, I825 and M844 side chains; the unsubstituted phenyl ring was trapped by pi-cation contacts with R847 and R850; the thiophene ring lay in a hydrophobic pocket mainly formed by R377, P378, N260 and N838 side chains; Furthermore, the thiophen aromatic ring behaved as weak H-bond acceptor [60] for the asparagine side chain amide moieties (Fig. 4). These interactions were stable through the simulation time confirming the stability of the proposed binding site.

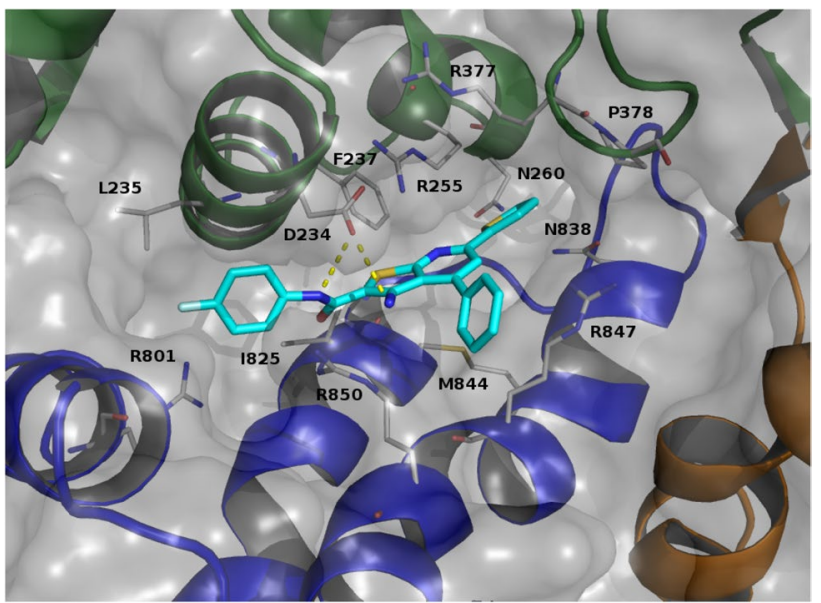

Fig. 4 Proposed binding mode for AM-001 (cyan). Epac1 is reported as cartoon: CNBD and DEP green; REMorange; CDC25-HD blue and RA red. Surface is reported in grey. Residues involved in interactions were depictedas grey stick. H-bond was reported as yellow dotted lines

With the aim to clarify the reason that were responsible for the selectivity of AM-001 between the two studied EPAC isoforms, we compared the two binding sites. The main difference deals with the residues of the loop between the CNBD b10 and REM b1. The loop was 19 residues long for EPAC1 and 18 residues long for EPAC2. Despite the similar length, just two residues were conserved between the two isoforms. Furthermore, the four residues facing the binding pocket for EPAC1(RPPT) were very different from the residues in the same positions of EPAC2 (NQGN). Thus, it may be argued that the behaviour of this loop in terms of conformation and interactions with AM-001 is responsible for the selectivity. Furthermore, R847 and I825, involved in contacts with AM-001 change to N (956) and T (934) respectively in EPAC2, weakening the binding stability. Also, D239 in EPAC1 correspond to E (345) in EPAC2. Taken together these data provide a possible explanation of the AM-001 selectivity toward EPAC1.

Then we docked the nine AM-001 analogues (Table S1) into this binding site, to evaluate the ability of the site to fit the experimental data [13]. The compounds AM-004 and AM-005 which differs just for the fluorine atom position had a very similar binding mode to AM-001, sharing the same interactions. For the compounds AM-003, AM-006, AM-007, AM-008 and AM-009 the binding mode was also comparable to AM-001, albeit with some evident differences: we did not observe the contacts of the fluorinated phenyl ring (AM-003 and AM-006), the thiophene (AM-007 and AM-008), and the unsubstituted phenyl ring (AM-009) of AM-001. For compound AM-002 we obtained a binding mode similar to AM-001, but we did not observe any $\mathrm{H}$-bonds, and the pyrimidone linked phenyl ring was place 


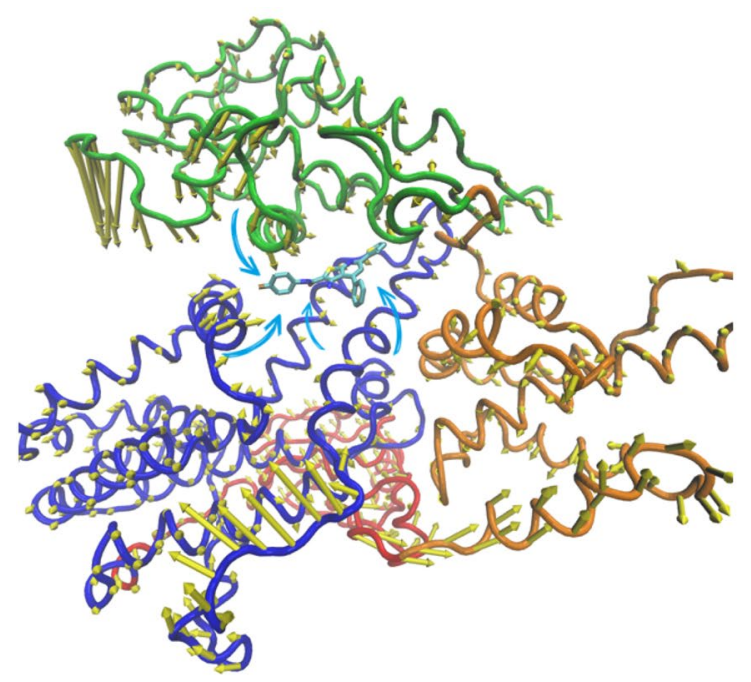

Fig. 5 Porcupine plot of the top two eigenvectors. Right panel: eigenvector 1, left panel: eigenvector 2 . Epac1 is reported as tube: CNBD and DEP green; REM orange; CDC25-HD blue and RA red. AM-001

father from the R801 (Fig. S10). Furthermore, we obtained a good correlation between the computed binding energy for the docking poses $[30,47]$ and the experimental biological activity with a significant correlation coefficient, $\mathrm{R}$, of 0.74 and Rs of $0.83 p$ (2-tailed) $0.53 \%$ (Table S2). The selected binding mode reasonably fits the SAR for AM-001 analogues. In conclusion, the selected binding mode reasonably fits the published structure-activity relationship for the AM-001 analogues, and seems suitable as a selective allosteric binding site for the inhibitor AM-001.

Lastly, we analysed the EPAC1/AM-001 complex trajectory with the aim of understanding how the AM-001 binding impaired the activating transition of the enzyme. PCA analysis was carried out to highlight the dominants mode of motion of the protein during the simulation time [61-63]. These protein motions were visualized by porcupine plots $[64,65]$ (Fig. 5) that show the direction and magnitude of the top two eigenvectors (Fig. S12) for each of the backbone $\mathrm{Ca}$ atoms. The most prominent observed motion was related to the loops at the CDC25-HD. Focusing on the helices that shaped the binding site we observed that a7 (794-805), a8 (821-830) and a9 (840-850) helices of the CDC25-HD moved toward the CNBD, as well as the CNBD a2 (230-242) moved toward the CDC25-HD.

The observed movements involved the enzyme loop and helices that are crucial in the open -to-close conformational transition. Thus, AM-001 binding to area 6 in the closed conformation of EPAC1 might strengthen the contact between the CDC25-HD and the CNBD domains, stabilizing an inactive-like conformation and preventing the activation transition otherwise induced by cAMP binding to the CNBD domain.

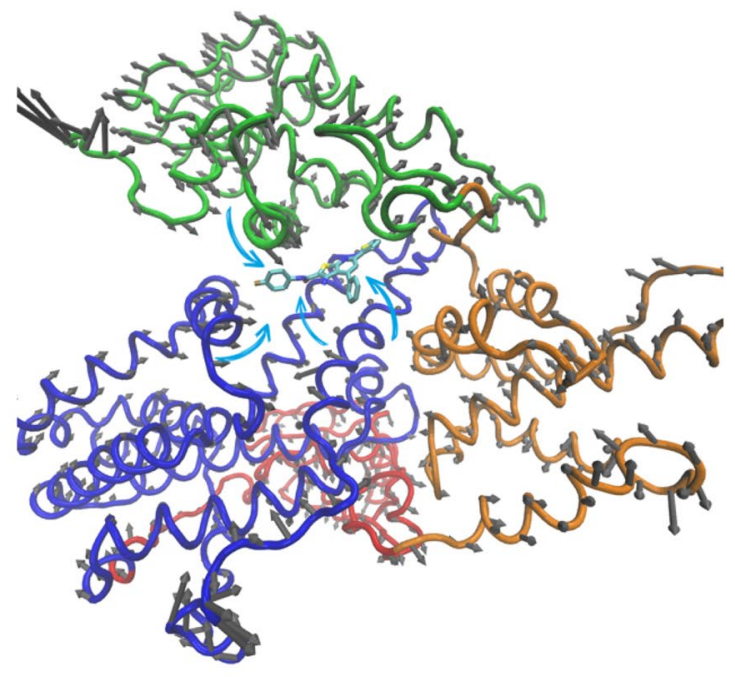

is reported as cyan stick. The yellow and grey arrows attached to each a-carbon atom indicate the direction of the movement; the size of each arrow shows the magnitude of the corresponding movement

\section{Conclusions}

Cosolvent molecular dynamics were performed with the aim to characterize a putative binding site for the EPAC1 allosteric inhibitor AM-001. By the cosolvent occupancy maps analyses we identified the EPAC1 most suitable sites for a partner binding. Each site was evaluated by the docking and molecular dynamics of AM-001. The pocket located at the interface between a8, a9 and a10 of CDC25HD and $\mathrm{a} 2$ and $\mathrm{a} 3$ of CNBD seems suitable as a selective allosteric binding site for the AM-001. The available AM-001 analogues were docked to this site showing a good match with the already reported SAR [13]. The PCA analyses of the EPAC1/AM-001 trajectory highlighted that the AM-001 binding might strengthen the contact between CDC25-HD of the catalytic region and CNBD of the regulatory region stabilizing an inactive "like" conformation. Thus, AM-001 binding to the predicted binding pocket may constrain the highly dynamic EPAC1 in an inhibited conformation, despite the binding of cAMP.

Acknowledgements Open access funding provided by Universit $\tilde{A}$ degli Studi di Roma La Sapienza within the CRUI-CARE Agreement.

Funding This work was supported by Excellence Departments grant from the Italian Ministry of Education, University and Research (MIUR, Italy) (Art. 1, commi 314-337 Legge 232/2016) to the Department of Drug Chemistry and Technologies (M.B., M.N.; R.S., and A.C.). 


\section{Compliance with ethical standards}

Conflict of interest The authors declare that they have no conflict of interest.

Open Access This article is licensed under a Creative Commons Attribution 4.0 International License, which permits use, sharing, adaptation, distribution and reproduction in any medium or format, as long as you give appropriate credit to the original author(s) and the source, provide a link to the Creative Commons licence, and indicate if changes were made. The images or other third party material in this article are included in the article's Creative Commons licence, unless indicated otherwise in a credit line to the material. If material is not included in the article's Creative Commons licence and your intended use is not permitted by statutory regulation or exceeds the permitted use, you will need to obtain permission directly from the copyright holder. To view a copy of this licence, visit http://creativecommons.org/licenses/by/4.0/.

\section{References}

1. Beavo JA, Brunton LL (2002) Cyclic nucleotide researchstill expanding after half a century. Nat Rev Mol Cell Biol 3:710-718

2. Schmidt M, Dekker FJ, Maarsingh H (2013) Exchange protein directly activated by cAMP (epac): a multidomain cAMP mediator in the regulation of diverse biological functions. Pharmacol Rev 65:670-709

3. Robichaux WG, Cheng X (2018) Intracellular cAMP Sensor EPAC: physiology, pathophysiology, and therapeutics development. Physiol Rev 98:919-1053

4. Niimura M, Miki T, Shibasaki T, Fujimoto W, Iwanaga T, Seino S (2009) Critical role of the N-terminal cyclic AMP-binding domain of Epac2 in its sub-cellular localization and function. $\mathrm{J}$ Cell Physiol 219:652-658

5. de Rooij J, Rehmann H, van Triest M, Cool RH, Wittinghofer A, Bos JL (2000) Mechanism of regulation of the Epac family of cAMP-dependent RapGEFs. J Biol Chem 275:20829-20836

6. Rehmann H, Arias-Palomo E, Hadders MA, Schwede F, Llorca O, Bos JL (2008) Structure of Epac2 in complex with a cyclic AMP analogue and RAP1B. Nature 455:124-127

7. Selvaratnam R, Mazhab-Jafari MT, Das R, Melacini G (2012) The auto-inhibitory role of the EPAC hinge helix as mapped by NMR. PLoS ONE 7:e48707

8. VanSchouwen B, Selvaratnam R, Fogolari F, Melacini G (2011) Role of dynamics in the autoinhibition and activation of the exchange protein directly activated by cyclic AMP (EPAC). J Biol Chem 286:42655-42669

9. Mazhab-Jafari MT, Das R, Fotheringham SA, Sildas S, Chowdhury S, Melacini G (2007) Understanding cAMP-dependent allostery by NMR spectroscopy: comparative analysis of the EPAC1 cAMP-binding domain in its apo and cAMP-bound states. J Am Chem Soc 129:14482-14492

10. Rehmann H, Prakash B, Wolf E, Rueppel A, de Rooij J, Bos JL, Wittinghofer A (2003) Structure and regulation of the cAMPbinding domains of Epac2. Nat Struct Biol 10:26-32

11. Bers DM (2008) Calcium cycling and signaling in cardiac myocytes. Annu Rev Physiol 70:23-49

12. El-Armouche A, Eschenhagen T (2009) $\beta$-adrenergic stimulation and myocardial function in the failing heart. Heart Fail Rev $14: 225-241$

13. Laudette M, Coluccia A, Sainte-Marie Y, Solari A, Fazal L, Sicard P, Silvestri R, Mialet-Perez J, Pons S, Ghaleh B, Blondeau JP, Lezoualc'h F (2019) Identification of a pharmacological inhibitor of Epac1 that protects the heart against acute and chronic models of cardiac stress. Cardiovasc Res 115:1766-1777

14. Bouvet M, Blondeau JP, LF (2019) The Epac1 protein: pharmacological modulators, cardiac signalosome and pathophysiology. Cells 8:1543-1561

15. Berman HM, Ten Eyck LF, Goodsell DS, Haste NM, Kornev A, Taylor SS (2005) The cAMP binding domain: an ancient signaling module. PNAS 102:45-50

16. Westley AM, Westley J (1996) J Biol Chem 271:5347-5352

17. Courilleau D, Bisserier M, Jullian JC, Lucas A, Bouyssou P, Fischmeister R, Blondeau JP, Lezoualc'h F, (2012) Identification of a tetrahydroquinoline analog as a pharmacological inhibitor of the cAMP-binding protein Epac. J Biol Chem 287:44192-44202

18. Courilleau D, Bouyssou P, Fischmeister R, Lezoualc'h F, Blondeau JP (2013) The (R)-enantiomer of CE3F4 is a preferential inhibitor of human exchange protein directly activated by cyclic AMP isoform 1 (Epac1). Biochem Biophys Res Commun 440:443-448

19. Tsalkova T, Blumenthal DK, Mei FC, White MA, Cheng X (2009) Mechanism of Epac activation: structural and functional analyses of Epac2 hinge mutants with constitutive and reduced activities. J Biol Chem 284:23644-23651

20. Brown LM, Rogers KE, Aroonsakool N, McCammon LA, Insel PA (2014) Allosteric inhibition of EPAC: computational modelling and experimental validation to identify allosteric site and inhibitors. J Biol Chem 289:29148-29157

21. Ghanakota P, Carlson HA (2016) Driving Structure based drug discovery through cosolvents molecular dynamics. J Med Chem 59:10383-10399

22. Allen KN, Bellamacina CR, Ding X, Jeffery CJ, Mattos C, Petsko GA, Ringe D (1996) An experimental approach to mapping the binding surfaces of crystalline proteins. J Phys Chem 100:2605-2611

23. Uehara S, Tanaka S (2017) Cosolvent-based molecular dynamics for ensemble docking: practical method for generating druggable protein conformation. J Chem Inf Model 57:742-756

24. Yu W, Lakkaraju SK, Raman EP, Fang L, MacKerell AD (2015) Pharmacophore modeling using site-identification by ligand competitive saturation (SILCS) with multiple probe molecules. J Chem Inf Model 55:407-420

25. Lexa KW, Goh GB, Carlson HA (2014) Parameter choice matters: validating probe parameters for use in mixed-solvent simulations. J Chem Inf Model 54:2190-2199

26. Lakkaraju SK, Mbatia H, Hanscom M, Zhao Z, Wu J, Stoica B, MacKerell AD Jr, Faden AI, Xue F (2015) Cyclopropyl-containing positive allosteric modulators of metabotropic glutamate receptor subtype 5. Bioorg Med Chem Lett 25:2275-2279

27. Rehmann H, Das J, Knipscheer P, Wittinghofer A, Bos JL (2006) structure of the cyclic-AMP responsive exchange factor Epac2 in its auto-inhibited state. Nature 439:625-628

28. Bakan A, Nevins N, Lakdawala AS, Bahar I (2012) Druggability assessment of allosteric proteins by dynamics simulations in the presence of probe molecules. J Chem Theory Comput 8:2435-2447

29. Jacobson MP, Pincus DL, Rapp CS, Day TJF, Honig B, Shaw DE, Friesner RA (2004) A hierarchical approach to all-atom protein loop prediction. Proteins 55:351-367

30. Schrödinger Release 2018-1: Prime, Schrödinger, LLC, New York, NY, 2018

31. Sastry GM, Adzhigirey M, Day T, Annabhimoju R, Sherman W (2013) Protein and ligand preparation: parameters, protocols, and influence on virtual screening enrichments. J Comput Aided Mol Des 27:221-234

32. Källberg M, Wang H, Wang S, Peng J, Wang Z, Lu H, Xu J (2012) Template-based protein structure modeling using the RaptorX web server. Nat Protoc 7:1511-1522 
33. Ma J, Wang S, Zhao F, Xu J (2013) Protein threading using context-specific alignment potential. Bioinformatics 29:257-265

34. Case DA, Cheatham TE 3rd, Darden T, Gohlke H, Luo R, Merz KM Jr, Onufriev A, Simmerling C, Wang B, Woods RJ (2005) The Amber biomolecular simulation programs. J Comput Chem 26:1668-1688

35. Martínez L, Andrade R, Birgin EG, Martínez JM (2009) Packmol: A package for building initial configurations for molecular dynamics simulations. J Comput Chem 30:2157-2164

36. Pettersen EF, Goddard TD, Huang CC, Couch GS, Greenblatt DM, Meng EC, Ferrin TE (2004) UCSF Chimera-a visualization system for exploratory research and analysis. J Comput Chem 25:1605-1612

37. Pintilie GD, Zhang J, Goddard TD, Chiu W, Gossard DC (2010) Quantitative analysis of cryo-EM density map segmentation by watershed and scale-space filtering, and fitting of structures by alignment to regions. J Struct Biol 170:427-438

38. Wang J, Wang W, Kollman PA, Case DA (2006) Automatic atom type and bond type perception in molecular mechanical calculations. J Mol Graph Model 25:247-260

39. Wang J, Wolf RM, Caldwell JW, Kollman PA, Case DA (2004) Development and testing of a general amber force field. J Comput Chem 25:1157-1174

40. Roe DR, Cheatham TE 3rd (2013) PTRAJ and CPPTRAJ: software for processing and analysis of molecular dynamics trajectory data. J Chem Theory Comput 9:3084-3095

41. Bakan A, Meireles LM, Bahar I (2011) ProDy: protein dynamics inferred from theory and experiments. Bioinformatics 27:1575-1577

42. Bakan A, Dutta A, Mao W, Liu Y, Chennubhotla C, Lezon TR, Bahar I (2014) Evol and ProDy for bridging protein sequence evolution and structural dynamics. Bioinformatics 30:2681-2683

43. Humphrey W, Dalke A, Schulten K (1996) VMD_visual molecular dynamics. J Mol Graph 14:33-38

44. Jones G, Willett P, Glen RC, Leach AR, Taylor R (1997) development and validation of a genetic algorithm for flexible docking. J Mol Biol 267:727-748

45. Korb O, Stutzle T, Exner TE (2009) Empirical scoring function for advanced protein-ligand docking with PLANTS. J Chem Inf Model 49:84-96

46. Morris GM, Huey R, Lindstrom W, Sanner MF, Belew RK, Goodsell DS, Olson AJ (2009) Autodock4 and AutoDockTools4: automated docking with selective receptor flexibility. J Comput Chem $16: 2785-2791$

47. Jacobson MP, Friesner RA, Xiang Z, Honig B (2002) On the role of crystal packing forces in determining protein sidechain conformations. J Mol Biol 320:597-608

48. The PyMOL Molecular Graphics System, Version 2.0 Schrödinger, LLC

49. Rehmann H, Selective activation of Epac1 and Epac2. To be published. (PDB code: 4MGI)

50. Peng W, Xu J, Guan X, Sun Y, Zhang X, Li X, Rao Z (2011) Structural study of the Cdc25 domain from Ral-specific guaninenucleotide exchange factor RalGPS1a. Protein Cell 2:308-319

51. Baameur F, Singhmar P, Zhou Y, Hancock JF, Cheng X, Heijnen CJ, Kavelaars A (2016) Epac1 interacts with importin $\beta 1$ and control neurite outgrowth independently of the cAMPc and Rap1. Sci Rep 6:6370-6381

52. Parnell E, Smith BO, Yarwood SJ (2015) The cAMPc sensors, EPAC1 and EPAC2, display distinct subcellular distribution despite sharing a common nuclear pore localization signal. Cell Signal 27:989-996

53. Mei FC, Cheng X (2005) Interplay between exchange protein directly activated by cAMP (Epac) and microtubule cytoskeleton. Mol BioSyst 1:325-331

54. Campbell SJ, Gold ND, Jackson RM, Westhead DR (2003) Ligand binding: Functional site location, similarity and docking. Curr Opin Struct Biol 13:389-395

55. Guo Z, Li B, Cheng LT, Zhou S, McCammon JA, Che J (2015) Identification Of Protein-Ligand Binding Sites By The Level-Set Variational Implicit-Solvent Approach. J Chem Theory Comput 11:753-765

56. Wells JA, McClendon CL (2007) Reaching for high-hanging fruit in drug discovery at protein-protein interfaces. Nature 450:1001-1009

57. Bahadur RP, Chakrabarti P, Rodier F, Janin JA (2004) Dissection of specific and non-specific protein-protein interfaces. J Mol Biol 336:943-955

58. Cui Q, Sulea T, Schrag JD, Munger C, Hung MN, Naïm M, Cygler M, Purisima EO (2008) Molecular dynamics and solvated interaction energy studies of protein-protein interactions: the MP1-p14 scaffolding complex. J Mol Biol 379:787-802

59. Naïm M, Bhat S, Rankin KN, Dennis S, Chowdhury SF, Siddiqi I, Drabik P, Sulea T, Bayly C, Jakalian A, Purisima EO (2007) Solvated interaction energy (SIE) for scoring protein-ligand binding affinities. 1. Exploring the parameter space. J Chem Inf Model 47:122-133

60. Kumar S, Das A (2012) Effect of acceptor heteroatoms on $\pi$-hydrogen bonding interactions: a study of indole $\cdots$ thiophene heterodimer in a supersonic jet. J Chem Phys 7,137(9):094309

61. Amadei A, Linssen AB, Berendsen HJ (1993) Essential dynamics of proteins. Proteins 17:412-425

62. Amadei A, Linssen AB, de Groot BL, van Aalten DM, Berendsen HJ (1996) An efficient method for sampling the essential subspace of proteins. J Biomol Struct Dyn 13:615-625

63. Yamaguchi H, van Aalten DM, Pinak M, Furukawa A, Osman R (1998) Essential dynamics of DNA containing a cis.syn cyclobutane thymine dimer lesion. Nucleic Acids Res 26:1939-1946

64. Tai K, Shen T, Borjesson U, Philippopoulos M, McCammon JA (2001) Analysis of a 10-ns molecular dynamics simulation of mouse acetylcholinesterase. Biophys J 81:715-724

65. Tai K, Shen T, Henchman RH, Bourne Y, Marchot P, McCammon JA (2002) Mechanism of acetylcholinesterase inhibition byfasciculin: a 5-ns molecular dynamics simulation. J Am Chem Soc 124:6153-6161

Publisher's Note Springer Nature remains neutral with regard to jurisdictional claims in published maps and institutional affiliations. 